\title{
Translating Evidence Into Impact: Research and Analyses for Scientific Transformation and Advancement (RASTA)
}

Population Council

Follow this and additional works at: https://knowledgecommons.popcouncil.org/

series_newsletters_researchimpact

How does access to this work benefit you? Let us know!

\section{Recommended Citation}

Population Council. 2021. "Translating Evidence Into Impact: Research and Analyses for Scientific Transformation and Advancement (RASTA)," Research Utilization and Impact Brief. Washington, DC: Population Council. 


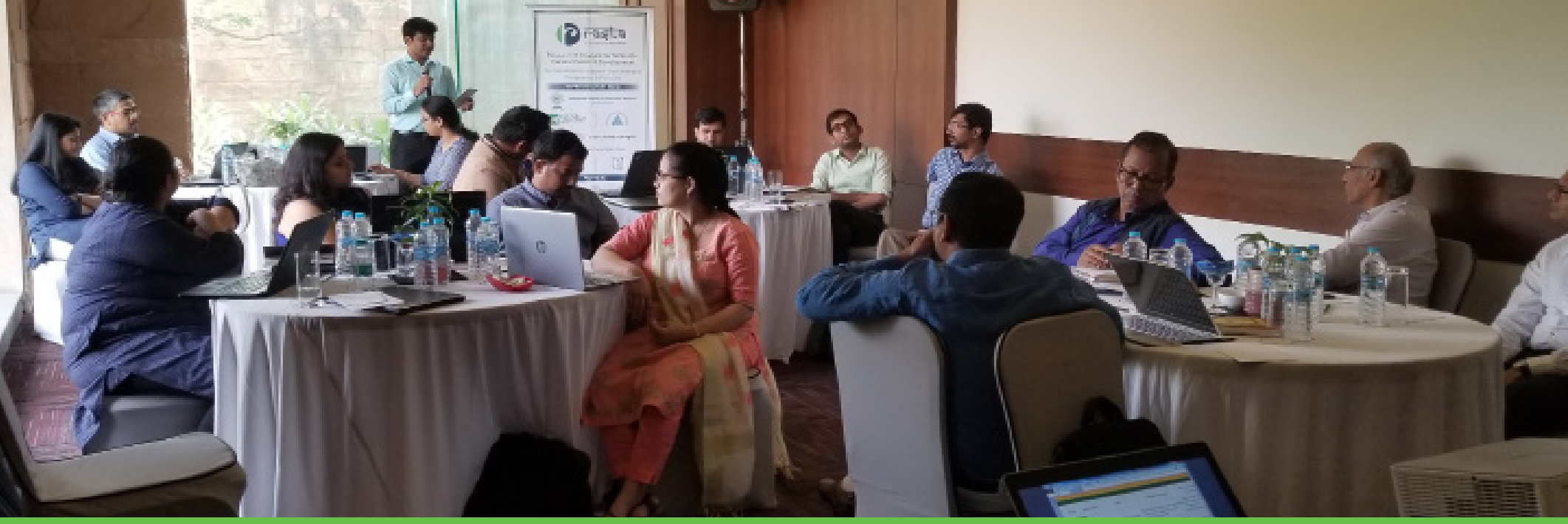

\section{Translating Evidence into Impact: Research and Analyses for Scientific Transformation and Advancement (RASTA)}

Summary: Since its creation in 2017, RASTA's collaborative research platform has continued to evolve and grow, promoting evidence use, enhancing coordination across a range of partners, and strengthening analytical skills of young Indian researchers to advance family planning and reproductive health (FP/RH). With a large body of research guided by questions developed in close collaboration with decision makers from government and other key stakeholders, RASTA has helped to fill critical evidence gaps and inform program, policy, and investment decisions, including around adolescent health and FP/RH access in the context of COVID-19.

Home to the second largest population globally, India has an abundance of health-related data and stakeholders working to support family planning and reproductive health (FP/RH); maternal, neonatal, and child health ( $\mathrm{MNCH}$ ); and adolescent health. However, available large data sets, such as the National Family Health Survey (NFHS), remain underutilized in shaping programs, policies, and investment decisions due to limited capacity for robust analysis and interpretation among various stakeholders and organizations. There is also a perennial need to continue to strengthen coordination and collaboration among the many stakeholders invested in improving FP/RH, MNCH, and adolescent health, including harmonization of data-driven program and policy recommendations.

To respond to this context, the Population Council convened RASTA, a consortium of institutions and individuals that aims to support government and non-governmental organizations with analyses and evidence for program strengthening on population, health, and development. A highly collaborative endeavor, RASTA has sought to utilize available large-scale data to respond to relevant program and policy questions, enhancing in-country capacities on analytics and program-oriented research in the process.

\section{OPERATING MODEL \& KEY ACTIVITIES}

The RASTA consortium brings together researchers from Indian academic institutions, as well as development partners and other collaborating organizations, to support evidence-informed programming and policymaking, particularly by the Ministry of Health and Family Welfare (MOHFW), 
Government of India. RASTA has employed several mechanisms to foster this close collaboration, such as creating a technical advisory group (TAG), co-convening joint meetings, sharing updates with government and donor partners, participating in other FP/RH consortia, and contributing to the national FP2020 dialogue.

These approaches have helped to grow the number of collaborating institutions, build a responsive research agenda, strengthen relationships among stakeholders, and enhance alignment of FP/RH messaging.

Novel Analysis: RASTA's consultative approach began with iteratively developing a list of key policy and program relevant research questions on FP/ $\mathrm{RH}$ with the TAG. From this, the consortium has carried out data mapping and analysis on these priority research questions in close partnership with researchers across India.
Findings have ascribed to five areas of analysis relevant to policy and programs: 1) policy, system, and market; 2) community-level; 3) household-level; 4) individual-level; and 5) data quality. RASTA has documented results through peer-reviewed articles, each accompanied by a policy brief to inform national and global FP/RH efforts.

\section{Capacity Building and Technical Assistance:} Across the continuum of research activities, RASTA has provided mentorship, capacity building, and technical assistance for early career researchers and interns. All research projects have involved at least one young researcher working under the supervision of a more experienced professor or expert. These young researchers were closely involved with defining research objectives, analytic design, data analysis and interpretation, and drafted and submitted articles to peer-reviewed journals.

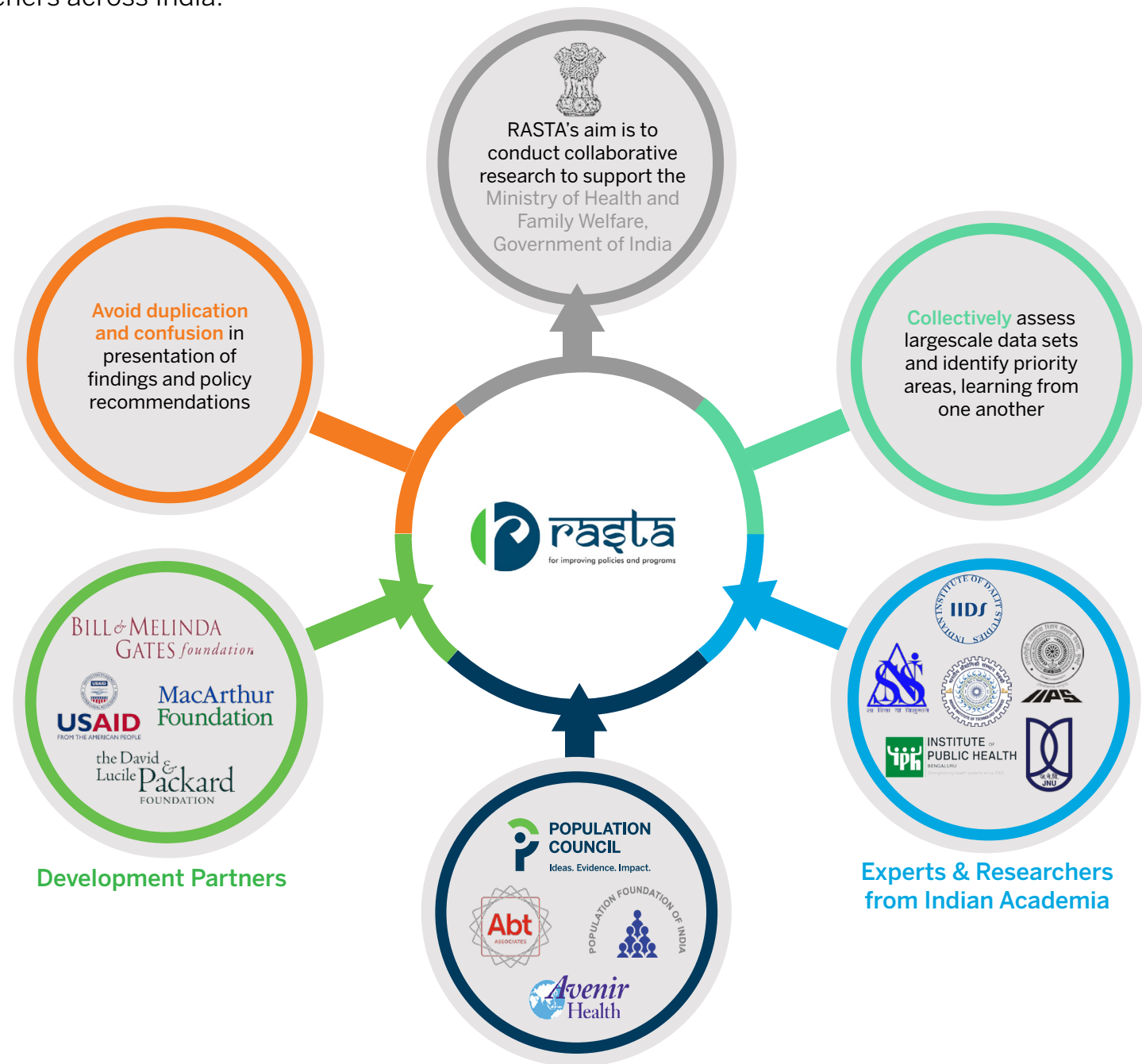

Collaborating Organizations 
In addition to direct mentorship, young researchers were provided additional technical assistance, including workshops on scientific writing and data analysis.

\section{RESEARCH IMPACTS}

Through a wide-ranging set of analyses and products featuring clear and tangible recommendations, the RASTA initiative has bolstered partners' data-driven decision-making at national and state levels.

Fostering Data-driven Programming through the Adolescent Health Data Tool

The Adolescent Health Data Tool was originally designed in close collaboration with the MoHFW to inform implementation of public-sector programs in India. It identifies multiple vulnerabilities in the domains of adolescent health defined by the national program Rashtriya Kishor Swasthya Karyakram using data from several existing sources, including the NFHS, National Census, and other sub-national data sets. It also provides information on the availability of resources at the district level, such as the density of health facilities per 1,000 adolescents. By providing comprehensive and accessible information on healthrelated vulnerabilities and resource availability in one place, the tool is tracking important indicators that can be used to understand adolescent health and

\section{RASTA by the Numbers: FP/RH Evidence \& Mentorship}

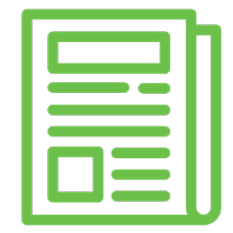

\author{
17 \\ Peer-reviewed \\ publications generated
}
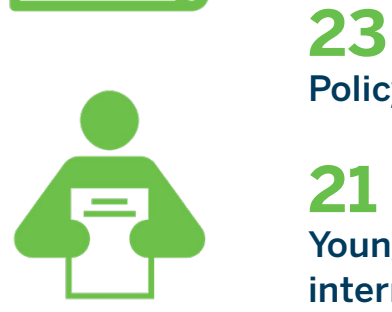

Policy briefs published

21

Young scholars and interns mentored in the research process

target programs accordingly. RASTA's national and state-level analyses have been used in consultations with national and state governments around adolescent program design.

\section{Understanding and Responding to FP/RH Needs in the Context of COVID-19}

Informed by conversations with several donor partners and stakeholders, RASTA was among the first to conduct research to understand how rapid implementation of COVID-19 lockdowns was impacting important FP/RH-related issues. Initial studies focused on unintended pregnancy, method discontinuation, and migration and FP use.

\section{Informing National \& Sub-National FP/RH Program \& Funding Decisions}

RASTA has catalyzed evidence-informed program, policy, and investment decisions to advance India's FP2020 agenda. By conducting new analyses and collaborative research responsive to questions identified by government and development partners, RASTA has filled data gaps to inform program and funding decisions at national and sub-national levels, including:

- Donor Program Strategy: Several development partners including the Bill and Melinda Gates Foundation, USAID, and WHO/India utilized RASTA analyses to inform their India FP and maternal, neonatal, and child health $(\mathrm{MNCH})$ program strategies.

- Packard Foundation Investment Addressing Migration and FP: RASTA evidence from Bihar examining migration and FP initiated discussions at state-level for programming with migrants and supported action to address the contraceptive needs of migrant households by state government during COVID-19.

- India's FP2020 Priorities: RASTA has presented in FP2020 meetings, and at Government of India meetings with FP2020, in conversations around priorities and program responses, including FP measures and private sector engagement. 
Conducted first in rural and peri-rural areas, early results led to the addition of urban districts across nine states, as well as questions focused on pregnancy intention and internet sourcing of contraceptives. Findings were shared in various meetings, including at meetings of the Advocating Reproductive Choices (ARC) collaborative, a consortium of civil society organizations who work on FP in India.

\section{LOOKING FORWARD}

As RASTA continues its work to enhance coordination, build capacity, and promote evidencebased decision making in $\mathrm{FP} / \mathrm{RH}, \mathrm{MNCH}$, and adolescent health, there is great interest surrounding this year's NFHS-5 data release. Updated analyses, based on government and stakeholder needs, will continue to inform conversations on issues such as contraceptive access among young users, expanding method choice, and reducing discontinuation.
There are plans to provide district-level comparisons of various indicators using NFHS-4 and NFHS5 data to better understand progress made and remaining gaps, and RASTA intends to provide in-depth analyses on several focus areas including $\mathrm{FP}, \mathrm{MNCH}$, adolescent health, tuberculosis, and noncommunicable diseases. This new data will also help inform RASTA's ongoing contributions to national and global discussions around India's FP2020/30 progress and commitments.

There is further interest in continuing to strengthen RASTA's state-level support. This will leverage established capacity strengthening and technical assistance approaches to support novel analyses by epidemiologists and statisticians, as well as new RASTA-supported research efforts and tools. Finally, RASTA seeks to expand collaborative analyses to support Government in achieving the Sustainable Development Goals.

To learn more, please refer to the following example resources:

\begin{tabular}{|c|c|}
\hline $\begin{array}{l}\text { Adolescent } \\
\text { Health Data } \\
\text { Tool }\end{array}$ & $\begin{array}{l}\text { - Brief: National analysis } \\
\text { - Brief: State-level analysis } \\
\text { - Brief: Union Territory analysis }\end{array}$ \\
\hline $\begin{array}{l}\text { FP use during } \\
\text { COVID-19 }\end{array}$ & $\begin{array}{l}\text { - Brief: Current and Unintended Pregnancy in Oct-Nov } 2020 \\
\text { - Brief: Migration and Family Planning } \\
\text { - Brief: Family Planning Service Delivery by Frontline Workers (FLWs) During April- } \\
\text { June } 2020 \\
\text { - Brief: Contraceptive Use and Methods Discontinuation Among Women Aged 15-34 } \\
\text { Years During March-November } 2020\end{array}$ \\
\hline
\end{tabular}

The Evidence Project is made possible by the generous support of the American people through the United States Agency for International Development (USAID) under the terms of cooperative agreement no. AID-OAA-A-13-00087. The contents of this document are the sole responsibility of the Evidence Project and Population Council and do not necessarily reflect the views of USAID or the United States Government.

The Population Council collaborates with program implementers, policymakers, researchers, and funding partners to advance evidence-based solutions to critical health and development challenges. Its research utilization and impact briefs feature recent highlights of our ongoing work to ensure evidence is translated into meaningful actions to improve lives from community to national, regional, and global levels.

Recommended citation: Population Council. "Translating Evidence into Impact: Research and Analyses for Scientific Transformation and Advancement (RASTA) ." Research Utilization and Impact Brief. Washington, D.C.: Population Council, 2021.

POPULATION COUNCIL

Ideas. Evidence. Impact. 